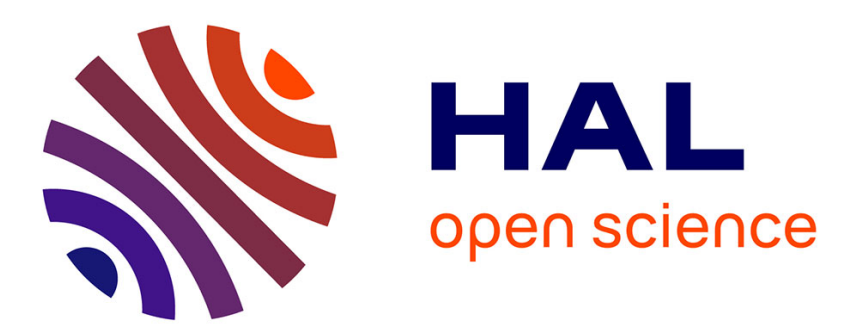

\title{
Mobile relay for LTE: proof of concept and performance measurements
}

\author{
Tanguy Kerdoncuff, Thomas Galezowski, Xavier Lagrange
}

\section{To cite this version:}

Tanguy Kerdoncuff, Thomas Galezowski, Xavier Lagrange. Mobile relay for LTE: proof of concept and performance measurements. VTC Spring 2018: IEEE 87th Vehicular Technology Conference, IEEE, Jun 2018, Porto, Portugal. 10.1109/VTCSpring.2018.8417774 . hal-01846055

\section{HAL Id: hal-01846055 \\ https://imt-atlantique.hal.science/hal-01846055}

Submitted on 20 Jul 2018

HAL is a multi-disciplinary open access archive for the deposit and dissemination of scientific research documents, whether they are published or not. The documents may come from teaching and research institutions in France or abroad, or from public or private research centers.
L'archive ouverte pluridisciplinaire $\mathbf{H A L}$, est destinée au dépôt et à la diffusion de documents scientifiques de niveau recherche, publiés ou non, émanant des établissements d'enseignement et de recherche français ou étrangers, des laboratoires publics ou privés. 


\title{
Mobile relay for LTE: proof of concept and performance measurements
}

\author{
Tanguy Kerdoncuff*, Thomas Galezowski ${ }^{\dagger}$, Xavier Lagrange* \\ *IMT Atlantique, IRISA, UBL, F-35576 Cesson Sévigné, France \\ $\dagger$ Société du Grand Paris, F-93200 Saint-Denis, France \\ *email: \{firstname.lastname\}@imt-atlantique.fr
}

\begin{abstract}
Even with dense base station deployments, public transport users often have a low quality for mobile services. Due to the insulation of the vehicle, passengers experience little to no connectivity on their end devices and low data bit rate. We thus propose a mobile relay architecture based on two imbricated levels of LTE networks and adapted to public transport systems. This architecture is evaluated on an actual LTE test-bed, consisting in standard radio interfaces, off-theshelf terminals and the Amarisoft software suite. One of the specifics of a mobile relay is the use of a radio link between the core network and the base stations, with all the limitations of this medium. This work therefore evaluates the impact on data rate of signaling mechanisms and of tunnelling in that context.
\end{abstract}

\section{INTRODUCTION}

The use of wireless broadband services is rising significantly with the deployment of Long Term Evolution (LTE) networks and the generalisation of smart phones, tablet computers and other new mobile devices. People make intensive use of these devices when they are on public transport vehicles such as buses, trams, or trains. It is anticipated that, by the year 2020, it will be fairly common to have up to 50 active vehicular User Equipments (UEs) per bus and up to 300 active vehicular UEs per train [1].

The service quality in public transport is far from satisfactory. Vehicles are usually well shielded with coated windows, which leads to a rather high penetration loss between outdoor and in-vehicle. The penetration loss can be as high as $25 \mathrm{~dB}$, and even goes up to $35 \mathrm{~dB}$ in Shanghai high-speed magnetic levitation train [2]. Traditionally, the UEs inside the public transport system are connected to macro base stations via wireless links, in which the penetration loss severely attenuates the signal quality and decreases the achievable data rate. Deploying mobile relays with both an outdoor antenna to communicate with the cellular network and an indoor antenna to provide a good coverage to passengers is a natural solution to provide a high Quality of Service (QoS).

Société du Grand Paris is in charge of designing and constructing the new $200 \mathrm{~km}$ of fully automated metro lines around Paris, connecting the 3 Paris airports as well as the main suburban and innovation areas. These "Grand Paris Express" lines, interconnected with existing Paris subway, regional trains and bus lines, will carry around 2 Million passengers per day. Société du Grand Paris is seeking to provide continuous high quality telecommunication services to passengers in Grand Paris Express stations and inside trains.
Thus, Société du Grand Paris is interested in developing new technologies such as mobile relays in order to serve this purpose.

Mobile relays have received a lot of attention from a theoretical point of view. In [3], the authors studied the performance of dual-hop transmission with a mobile relay and with a fixed relay by means of a theoretical analysis and proved the benefit of mobile relays when the penetration loss is above $25 \mathrm{~dB}$. In [4], stochastic analysis is used to compute the capacity of a cell when mobile relays are deployed. In [5] joint transmission by both a mobile relay and a macro-cell base station is proposed to improve the data rate of non-vehicular UEs and the outage probability is derived. All these publications focus on the transmission issues and have a theoretical approach.

Some studies consider the mobility protocol issues. In [6], several architectures based on the Third Generation Partnership Project (3GPP) analysis [7] are described. In [8], [9], the authors optimised handover for mobile relays deployed on high speed trains by using the predictability of the movement on a railway. In [10], the authors proposed to define "mobility areas" for mobile relays and analysed the handover latency when the mobile relay stays in the same mobility area or moves to another one. However, this proposal requires extra planning effort on the operator side and some upgrades of the standard 3GPP based protocols. To the best of our knowledge there is no paper about the performance of a system with a mobile relay with standard LTE interfaces.

3GPP investigated several possible architectures for mobile relays in [7]. Some solutions require the modification of several protocols and thus an important standardisation effort. However, the first alternative (called alt1) described in [7] is based on the fact that nodes of the Evolved Packet Core (EPC) such as eNBm, MME just need IP connectivity and that IP connectivity can be simply provided by an LTE/EPC network. The objective of this paper is to demonstrate that mobile relaying compliant with alt1 of 3GPP can be easily implemented with standard EPC nodes and to measure the performance of such a solution on a test-bed with real radio transmissions.

The paper is organised as follows. In section II we describe the architecture and the protocol stack when mobile relays are used. In section III we present our test-bed. In section IV, we measure the performance for a web-browsing service and in section $\mathrm{V}$, we study the impact of a mobile-relay handover on 


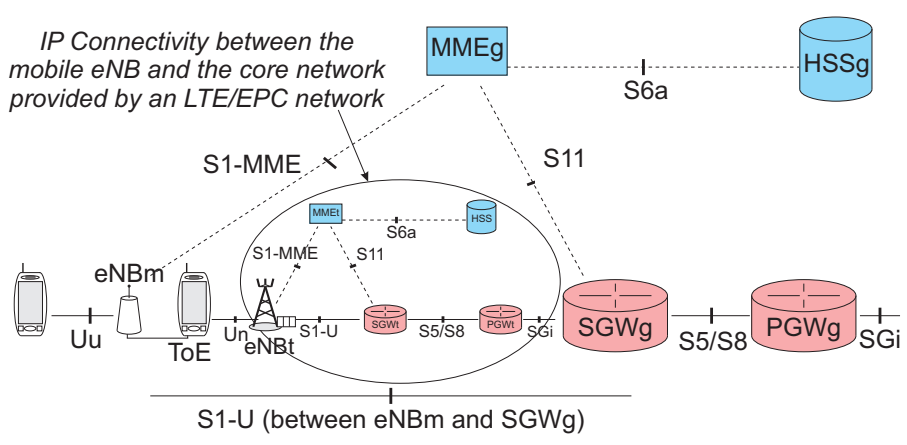

Fig. 1: Architecture of a network with a mobile relay

a data transfer. Section VI summarises our main findings.

\section{Mobile Relay Architecture}

An LTE/EPC mainly includes the following nodes: the Evolved Node B (eNB), the Mobile Management Entity (MME), Serving Gateway (SGW), Packet Gateway (PGW), and the Home Subscriber Server (HSS).

The wireless access for passengers is provided by the use of two LTE/EPC networks as shown in Figure 1. The first one is called the Track Evolved Packet Core (EPCt) and can be for example deployed by the transport operator. Nodes of this network are identified by suffix $\mathrm{t}$ (eNBt, MMEt, SGWt, etc.). We name Transport Operator terminal Equipment (TOE) a terminal of this network. There is typically one TOE in every train. The second network is the general network. It manages the UEs of passengers and is owned by the usual telecommunication operator. It has Mobile eNB (eNBm) as well as standard eNB. Note that eNBms have access to the general network through the track network: an eNBm is connected to a TOE and can thus exchange messages with Global Mobile Management Entity (MMEg) and Global Serving Gateway (SGWg).

It is worth mentioning that all these elements are standard LTE components, and that the use of this architecture requires no adaptation of protocol stacks or hardware.

There can be several general networks that use the same LTE/EPC track network in order to allow all mobile operators of a country to provide the service on board. There can be several eNBms (one per operator) but Radio Access Network (RAN) sharing can also be used to avoid a duplication of embedded hardware in trains. Note that Figure 1 shows a functional architecture. The operator of the track network can be a mobile operator and several entities can be physically in the same node (e.g. MMEt and MMEg are in the same piece of equipment)

The protocol stack when a mobile relay is used is shown in Figure 2 on the radio interface and on the S1-interface. Due to the use of two LTE/EPC networks there is an additional level of encapsulation and thus extra-headers compared to a standard architecture. The following headers are added to each packet: 8 bytes for GPRS Tunneling Protocol (GTP) [11], 8 bytes for User Datagram Protocol (UDP) and 20 bytes for

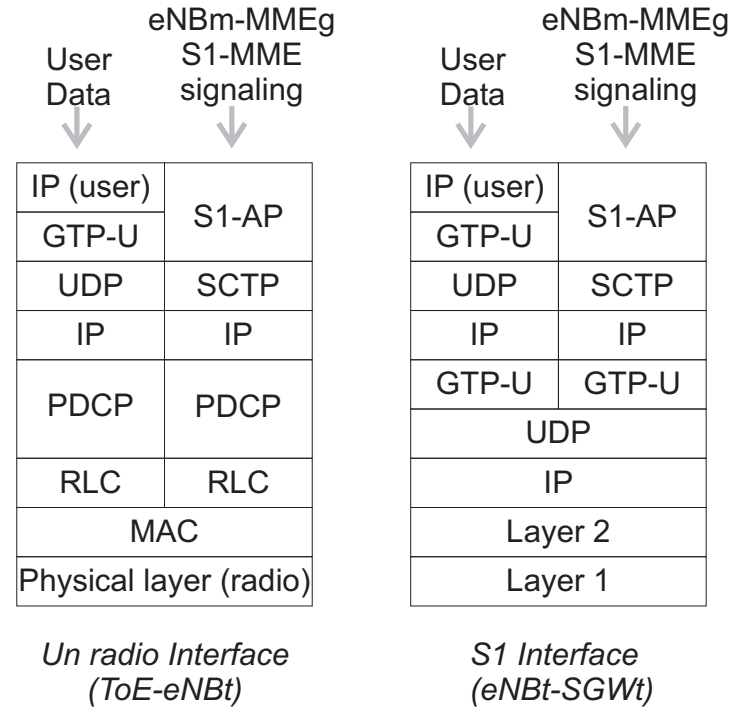

Fig. 2: Protocol stack with a mobile relay

Internet Protocol (IP) (with IPv4). The total length of this extra overhead is fixed but the length of packets is variable: an important question is to determine whether this 36-byte overhead is negligible or not compared to the user data average size for a popular service as web browsing.

One important benefit of mobile relay is that mobile management is no more done on an individual terminal basis but at the relay level. When a terminal is attached to the network through the relay, the terminal is considered as being under the coverage of the mobile eNB and is fixed as long as the user stays in the train. If the train moves to another cell, a single handover command is sent to the TOE but applies for all terminals connected to the mobile eNB. An interest of performing only one handover per train, instead of one handover per passenger can be found in the messages exchanged during a handover. For example, the message UECapabilitiesInformation describes the LTE capabilities of an UE and can be several 100-bytes long. Sending this message only once for the TOE (instead of as many times as the number of active terminals in the train) is a valuable argument in favour of a mobile relay. However, such a TOE handover still causes an interruption in the user data transmission because soft handover is not available in LTE/EPC. An important problem is to know whether such an interruption has a major impact on the TCP behaviour.

\section{AmARisoft LTE TestBed}

Our test-bed is based on the Amarisoft solution [12] which is a low-cost LTE base station and EPC core network running on a personal computer. Digital signal processing and the whole protocol stacks are pure software operations. We fixed the bandwidth of each radio interface to $5-\mathrm{MHz}$ in order to limit the processing load of the computer. The transmission is made in frequency division duplex mode in the $2.6 \mathrm{GHz}$ band. In order to avoid interference between the access and the backhaul links, two separate carriers are used. 


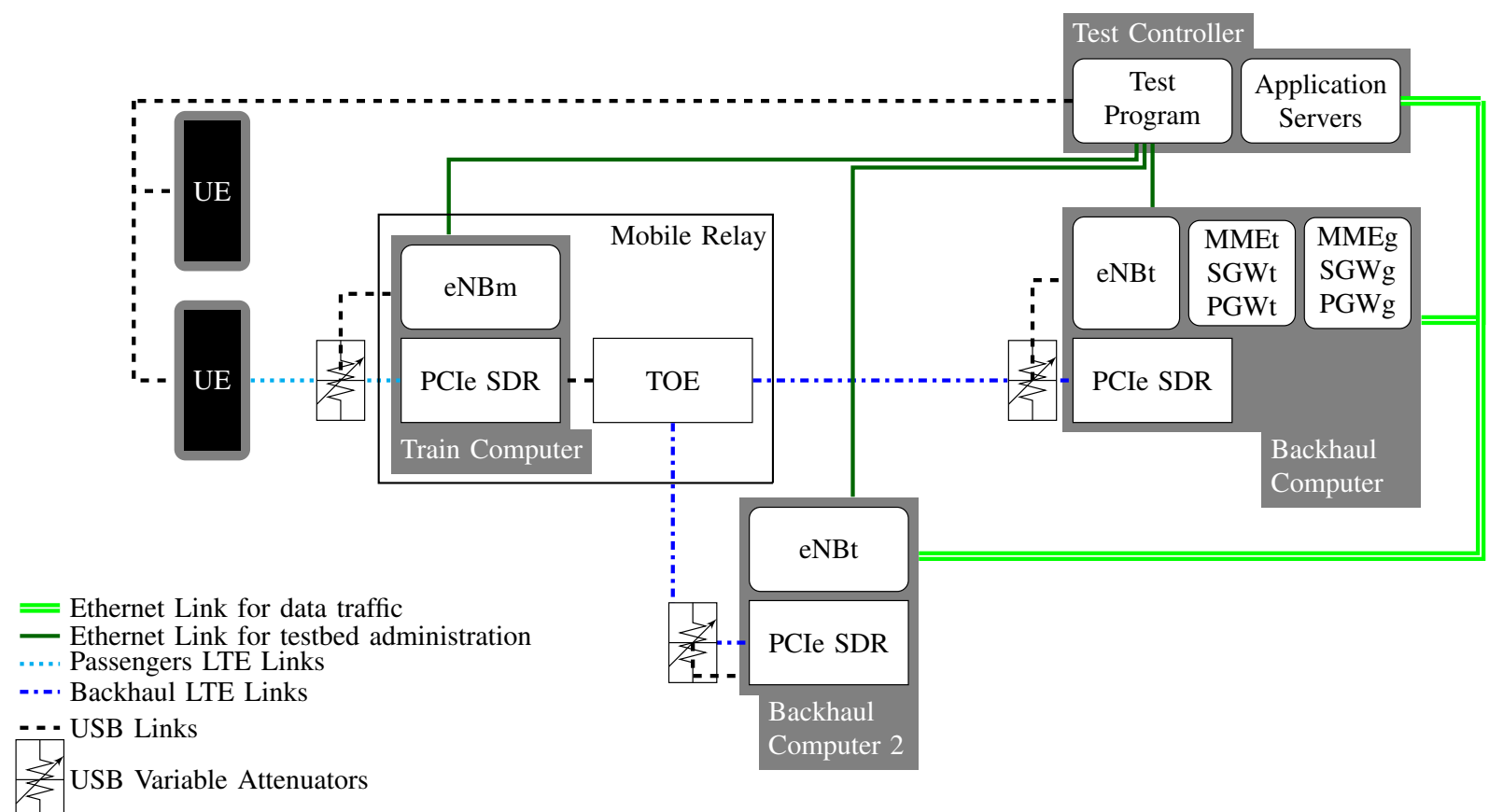

Fig. 3: Testbed Architecture

Figure 3 presents the layout of the test-bed, with its actors and connections. The four gray blocks represent the different physical machines that were involved in the experimental process, and are detailed below. The Test Controller is responsible for the coordination of all the other actors. It is connected to the other computers of the test-bed via Ethernet or to UEs via an USB link. It controls all other actors of the testbed, such as the amarisoft instances, the UEs or the USB variable attenuators, used to degrade or improve the quality of radio link and induce handovers.

Three instances of amarisoft programs run on the backhaul computer. First, the lteenb program manages the Track eNB (eNBt). Then, two separated instances of the ltemme program run independently to manage on one hand the Track Mobile Management Entity (MMEt), Track Serving Gateway (SGWt) and Track Packet Gateway (PGWt) functions used by the UE associated to the relay, and on another hand, the MMEg, SGWg and Global Packet Gateway (PGWg) required to handle the UEs. It is connected to a wired network, allowing it to route traffic from the UEs towards application servers located up link.

The Train Computer plays the role of the mobile relay running on-board the train. It is equipped with an lteenb amarisoft program to play the role of eNBm while the MMEg and PGWg functions are running in the backhaul computer. It is also equipped with an LTE USB dongle playing the role of the TOE.

In order to perform Relay Node (RN) handovers, backhaul computer 2 is required. It runs an instance of the amarisoft lteenb program to control its eNBt. That eNB connects by ethernet to the MMEt running on the first backhaul computer.

\section{DATA TRANSFER PERFORMANCES}

This section presents the performance achieved by UEs using our test-bed while loading web pages. Every year the french regulatory authority ARCEP measures the QoS of operational mobile networks. We based our tests on the 26 different web pages used in the 2016 ARCEP campaign [13]. The objective of this experiment is to check that web browsing is possible in a relay-based architecture with acceptable quality of service in non-overloaded conditions. A secondary objective is to measure the overhead due to multiple encapsulations and the amount of signaling on a practical implementation of the protocols.

The results presented in this section focus on four websites of the aforementioned ARCEP campaign, each one of them being opened by the UE's browser. The numbers we present in this work were obtained by realising 400 such occurrences for each site through our mobile relay test-bed, and 400 more times through a direct LTE connection that consists in a single eNB and EPC and that is called the "baseline".

Figure 4a presents the time it took for the UEs to retrieve $80 \%$ of the traffic observed after opening the web page. This avoids taking into account periodic traffic generated by a page's asynchronous elements such as ads. Comparing the distribution of direct LTE to the mobile relay setup gives us an estimation of the few additional hundred milliseconds it took the UE to load most of a page's elements, and by doing so, make it readable for the end user. Mobile relays being aimed at areas where such a direct LTE coverage is physically impossible, we believe this additional delay to be acceptable.

As explained in section II (see Figure 2), there is an additional tunnelling process when mobile relays are used. In most 


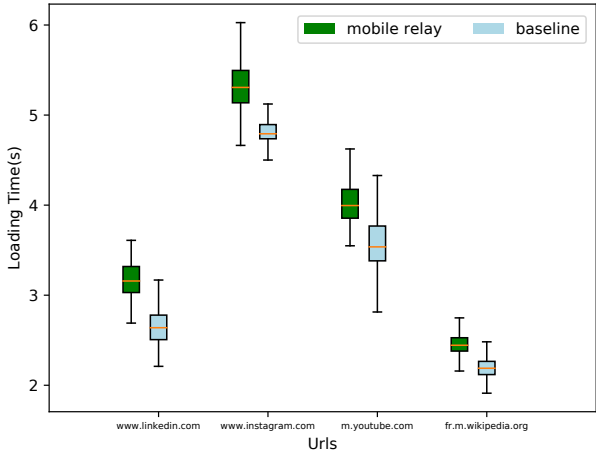

(a) HTTP Loading times $(80 \%)$

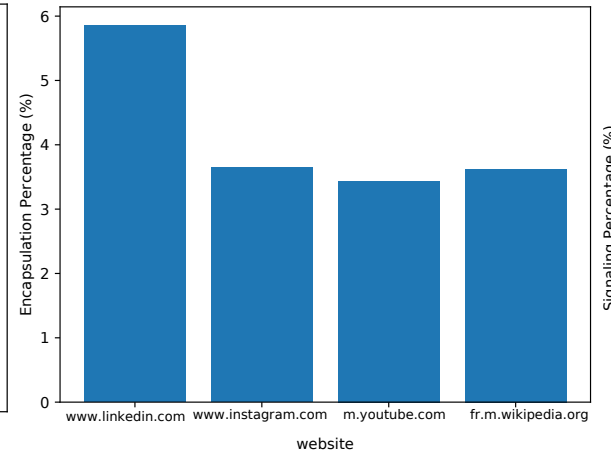

(b) LTE Encapsulation overhead

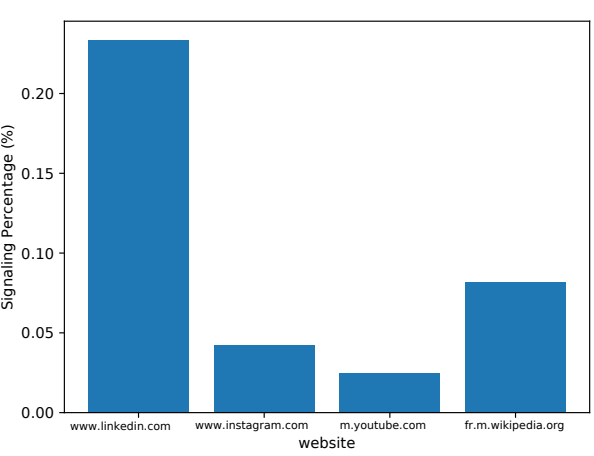

(c) LTE Signaling Overhead

Fig. 4: HTTP Transfer analysis

cases, extra headers have a low impact as shown in figure $4 \mathrm{~b}$ because packets are long enough: with payloads making the total size of packets close to the Maximum Transmission Unit (MTU) the extra header is at most $6 \%$ of the load when IPv4 is used. With IPv6, the extra-header is longer ( 56 bytes instead of 36 ), which can be roughly estimated as $\frac{56}{36} \times 6=9,3 \%$ of the load.

With mobile relays, some signaling messages between the eNBm and the Global Evolved Packet Core (EPCg) are transmitted on the backhaul radio link. However, figure 4c shows that these signaling messages are negligible compared to the amount of user data: less than $0.10 \%$ in most case. The difference between the first website and the other ones, is that it maintains inactive TCP connections after the page finished loading. Closing the browser, and therefore the TCP connections at the end of each experiment reactivates the sleeping LTE link, and therefore generates additional signaling.

In our experiments, all radio links are in line-of-sight conditions. Thus, our baseline does not represent the QoS experimented by users in a transport system. These results should not be read as a decrease of the quality of service when introducing a mobile relay, since without it there can be no or very degraded service. It should, on another hand, be read as the additional traffic to take into account when using this architecture to bring cellular coverage to new, previously uncovered, areas. For example, if a standard base station can accept a $6 \%$ increase in traffic due to encapsulation and signaling, it is then acceptable to use a mobile relay.

\section{RELAY NODE HANDOVER}

This section studies handovers of the TOE, which happens when the train moves between cells covered by different eNBt. This experiment allowed us to confirm that only one handover operation is executed and to check whether the temporary loss of connectivity and possible link quality change impacts the on board UEs.

To ensure the UEs were in connected state (in the LTE meaning of the word) during the experiment, an HTTP down link transfer is generated from the test controller. At a given time, the source eNBt instructs the TOE to switch to a

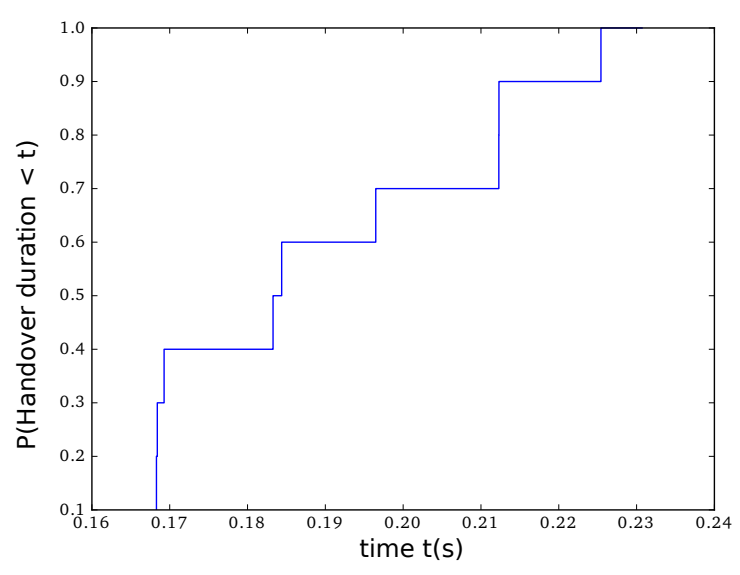

Fig. 5: Cumulative Distribution Function (CDF) of RN Handover duration from the eNBm standpoint

neighbouring cell. Traffic capture on both eNBt are performed during the handover, in order to locate signaling messages and the UE traffic on both base stations.

We define the handover duration from the eNBms standpoint as the duration between the sending of the LTE connection reconfiguration message on the source eNBt and the moment from which a packet can be transmitted by the relay to target eNBt. Figures 5 presents the Cumulative Distribution Function (CDF) of such handover duration. The maximal handover duration's is around $230 \mathrm{~ms}$, a value that remains small compared to, for example, the initial TCP re-transmission timer (1s) [14].

Another important aspect of this experiment is the confirmation that such handovers did not interfere in an unforeseen way with the train's passengers' experience : UEs are able to maintain traffic and LTE attachment without any perturbation.

Figure 6 presents the impact a TOE handover may have on an UE performing traffic. Its $\mathrm{X}$ axis marks time while the $\mathrm{Y}$ axis presents the cumulative amount of bytes the UE was able to download. The slope gives the throughput. Around $t=6 s$, we observe an area of non progress delimited by the 


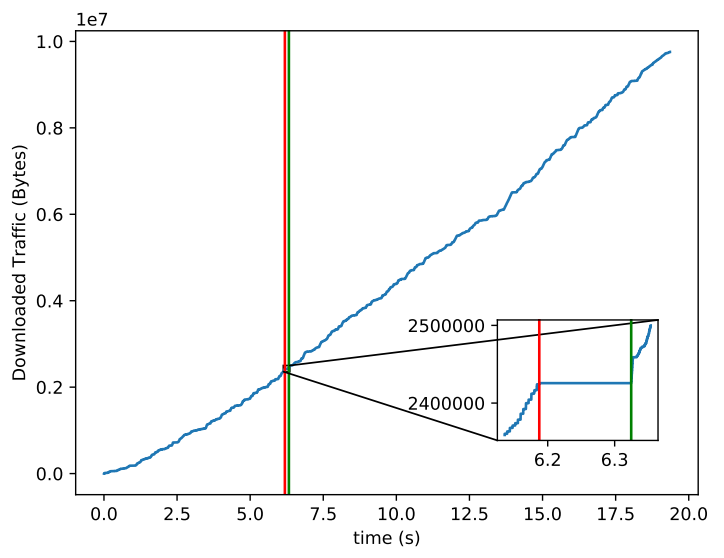

Fig. 6: Evolution of transmission and RN Handover impact on UE traffic (the vertical lines show the beginning and the end of the handover)

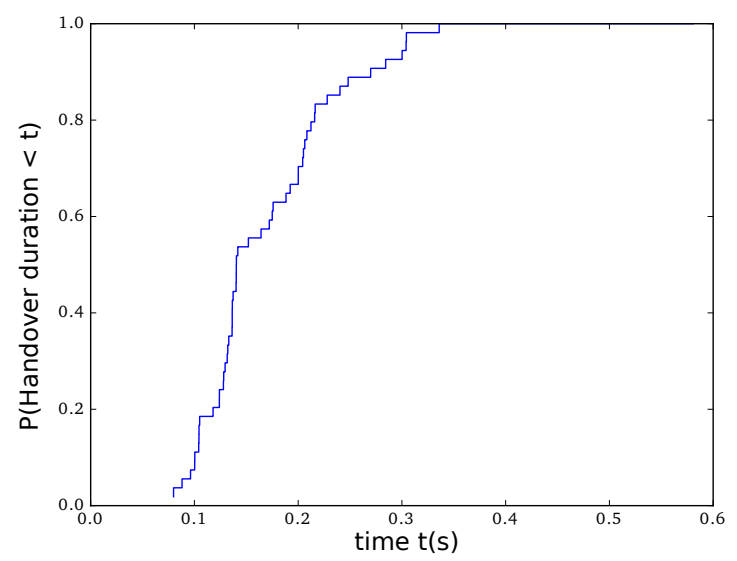

Fig. 7: Cumulative distribution of the duration of traffic interruption

two vertical lines. Though an RN handover is invisible from a signaling point of view to the UEs on-board the train, it is responsible for putting the traffic on hold during this period of time.

Figure 7 presents the $\mathrm{CDF}$ of the interruptions observed in UE traffic. In each case, the duration taken into account is the longest one observed during an experiment featuring a single $\mathrm{RN}$ handover. In $80 \%$ of the performed experiments, the longest interruption is less than $200 \mathrm{~ms}$, while the maximal observed duration is slightly over $300 \mathrm{~ms}$. It is important to emphasise that this graph needs to be read as an upper bound of the handover interruption duration, as other interruptions can happen independently from the handovers, as observable in Figure 6. Still, these values are in the range of interruptions that most mobile applications already deal with by, for example, content caching on the UE.

\section{CONCLUSION}

In this work, we proved that deploying mobile relays is possible in a standard LTE architecture. Using a testbed with off-the-shelf UEs, we evaluated the impact the different LTE mechanisms can have on a web service when cohabiting in a mobile relay setup. We found out that neither encapsulation nor signaling on a radio link was prohibitive. We also studied mobility, in particular the mobility of the eNBm to which the associated UEs are blind. We observed that, on one hand, such handovers were not disruptive, and on another hand, that they occurred under a reasonable amount of time.

In this test, only two terminals were used. A perspective is to study the behaviour of such a system when a train is full of passengers. Some additional simulations would be needed to test a full charged vehicle in a close to real field conditions.

\section{REFERENCES}

[1] P Popovski, V Braun, HP Mayer, P Fertl, Z Ren, D Gonzales-Serrano, E Ström, T Svensson, H Taoka, P Agyapong, et al. Scenarios, requirements and kpis for $5 \mathrm{~g}$ mobile and wireless systems. The METIS project: Mobile and wireless communications Enablers for the Twentytwenty Information Society, Tech. Rep. ICT-317669-METIS D, 1, 2013.

[2] Liu Liu, Cheng Tao, Jiahui Qiu, Houjin Chen, Li Yu, Weihui Dong, and Yao Yuan. Position-based modeling for wireless channel on high-speed railway under a viaduct at $2.35 \mathrm{ghz}$. IEEE Journal on Selected Areas in Communications, 30(4):834-845, 2012.

[3] Yutao Sui, Agisilaos Papadogiannis, and Tommy Svensson. The potential of moving relays-a performance analysis. In Vehicular Technology Conference (VTC Spring), 2012 IEEE 75th, pages 1-5. IEEE, 2012.

[4] Y. Chen, F. Yan, and X. Lagrange. Performance analysis of cellular networks with mobile relays under different modes. Telecommunication systems, 66(2):217-231, Oct 2017. Published on line 03 february 2017.

[5] Xiaoxuan Tang, Xiaodong Xu, Tommy Svensson, and Xiaofeng Tao. Coverage performance of joint transmission for moving relay enabled cellular networks in dense urban scenarios. IEEE Access, 5:1300113009, 2017.

[6] Lin Chen, Ying Huang, Feng Xie, Yin Gao, Li Chu, Haigang He, Yunfeng Li, Feng Liang, and Yifei Yuan. Mobile relay in 1te-advanced systems. IEEE Communications Magazine, 51(11):144-151, November 2013.

[7] 3rd Generation Partnership Project. Evolved Universal Terrestrial Radio Access (E-UTRA); Study on mobile relay (Release 12). V12.0.0 Technical Report 36.836, 3GPP, June 2014.

[8] Meng-Shiuan Pan, Tzu-Ming Lin, and Wen-Tsuen Chen. An enhanced handover scheme for mobile relays in lte-a high-speed rail networks. IEEE Transactions on Vehicular Technology, PP(99):1, 2014.

[9] Qing Huang, Jianmei Zhou, Cheng Tao, Su Yi, and Ming Lei. Mobile relay based fast handover scheme in high-speed mobile environment. In IEEE Vehicular Technology Conference (VTC Fall), pages 1-6, September 2012.

[10] Christian Pietsch, Stefan Brueck, Michael Farber, Thiago Moraes, Mourad Khanfouci, Jaakko Vihriala, M. Danish Nisar, Eiko Seidel, Tommy Svensson, Agisilaos Papadogiannis, Yutao Sui, and Mikael Sternad. Moving Relays and Mobility aspects. Technical report, Artist4G deliverable, May 2012.

[11] 3rd Generation Partnership Project. General packet radio system (gprs); tunnelling protocol user plane (gtpv1-u) (release 8). V8.5.0 Technical Specification 29.281, 3GPP, March 2010.

[12] Amarisoft. Amari lte 100, software lte base station on pc. Available at: http://www.amarisoft.com/.

[13] ARCEP. Enquete sur la qualité des services de données des opérateurs mobiles en france métropolitaine. Technical report, september 2016.

[14] V. Paxson, M. Allman, J. Chu, and M. Sargent. Computing tcp's retransmission timer. RFC 6298, RFC Editor, June 2011. 